\title{
Guillain-Barré syndrome associated with BNT162b2 COVID vaccination: a first case report from South Korea
}

\author{
Namkyun Kim ${ }^{1}$ · Jae-Hyung Kim ${ }^{2}$ · Jin-Sung Park ${ }^{2}$ (D)
}

Received: 8 December 2021 / Accepted: 20 December 2021 / Published online: 3 January 2022

(c) Fondazione Società Italiana di Neurologia 2022

\section{Dear Editor,}

Guillain-Barré syndrome (GBS) is an acute immune-mediated polyradiculoneuropathy usually triggered by antecedent events such as infection and vaccination. Since the global outbreak of coronavirus disease 2019 (COVID-19), as declared by the World Health Organization in March 2020, there are reports of GBS related to COVID-19 vaccination. Herein, we report, for the first time in South Korea, the case of a 21-year-old man who presented with facial diplegia and mild ataxia that later progressed to mild motor weakness after the first dose of BNT162b2 (Pfizer) vaccination.

The patient visited our hospital with bilateral facial weakness and paresthesia in both limbs and progressive lower extremity weakness after completion of BNT162b2 (Pfizer) vaccination 3 weeks ago (Fig. 1A). The initial neurologic examination showed bilateral facial diplegia with a motor grade weakness of $4+$ in the lower extremities. He showed areflexia in upper and lower extremities. His gait was ataxic, and a slight dysmetria was present in the upper extremities. He also noticed paresthesia in all extremities and complained of mild urinary and erectile dysfunction. The nerve conduction study was performed, and it showed a sural sparing pattern with mild prolongation of the $\mathrm{F}$ waves and decreased compound motor action potentials in the motor nerves without significant demyelinating features. The facial nerve conduction study revealed bilateral prolongation of $\mathrm{R} 1$ and R2, which were compatible with bilateral facial palsy. The heart rate variability threshold test showed a significant

Jin-Sung Park

neurojspark@gmail.com

1 Department of Internal Medicine, School of Medicine, Kyungpook National University, Kyungpook National University Hospital, Daegu, South Korea

2 Department of Neurology, School of Medicine, Kyungpook National University, Kyungpook National University Chilgok Hospital, Daegu, South Korea decrease in the blood pressure that was compatible with orthostatic hypotension.

Among the laboratory findings, the patient showed hyponatremia ( $\mathrm{Na}, 129 \mathrm{mmol} / \mathrm{L}$ ), and the cerebrospinal fluid study (CSF) showed albuminocytologic dissociation with an elevated protein level of $72.7 \mathrm{mg} / \mathrm{dL}$. CSF and serum laboratory findings including cytomegalovirus, Epstein-Barr virus, mycoplasma, mumps virus, measles virus, rubella, herpes simplex virus, and varicella-zoster virus IgM antibodies were negative. The human immunodeficiency virus antigen/antibody, cryptococcus antigen, and hepatitis labs were also negative. The Campylobacter jejuni antibody was also negative. Autoimmune-related laboratory tests such as rheumatoid factor, thyroid function test, SSA, SSB, antinuclear antibodies, antineutrophil cytoplasmic antibodies, lupus anticoagulant antibody, and anti-dsDNA were all unremarkable. Antiganglioside antibodies encompassing anti-GM1 IgG, anti-GQ1b IgG, anti-GT1a, and anti-GD1b IgG were also unremarkable. The patient received SARS$\mathrm{CoV}-2$ serology test, which was negative for IgM, IgG, and $\mathrm{Ig} \mathrm{A}$ antibodies to nucleocapsid protein.

The lumbar spine magnetic resonance imaging illustrated prominent enhancement in the nerve roots of the cauda equina that were radiologically consistent with GBS (Fig. 1B).

The young patient was diagnosed with GBS with prominent facial diplegia, and $2 \mathrm{~g}$ of intravenous immunoglobulin (IVIg) was administered for 5 consecutive days. The patient showed no complications related to treatment, and there was mild recovery after the therapy. After 1-month follow-up, the patient recovered from facial diplegia, ataxia, and paresthesia (Fig. 1C).

With the advent of COVID-19 respiratory disease that caused a pandemic in 2020, the Food and Drug Administration hastened the approval of four COVID-19 vaccines. Two of them are messenger RNA vaccines (BNT162b2, Pfizer BioNtech; mRNA-1273, Moderna), and the other two are adenovirus vector-based vaccines (Ad26.COV2.S, Johnson 
Fig. 1 The patient presented with facial diplegia (A) with cauda equine enhancement in the lumbar magnetic resonance imaging (B). Facial diplegia recovered after 1 month of treatment $(\mathbf{C})$

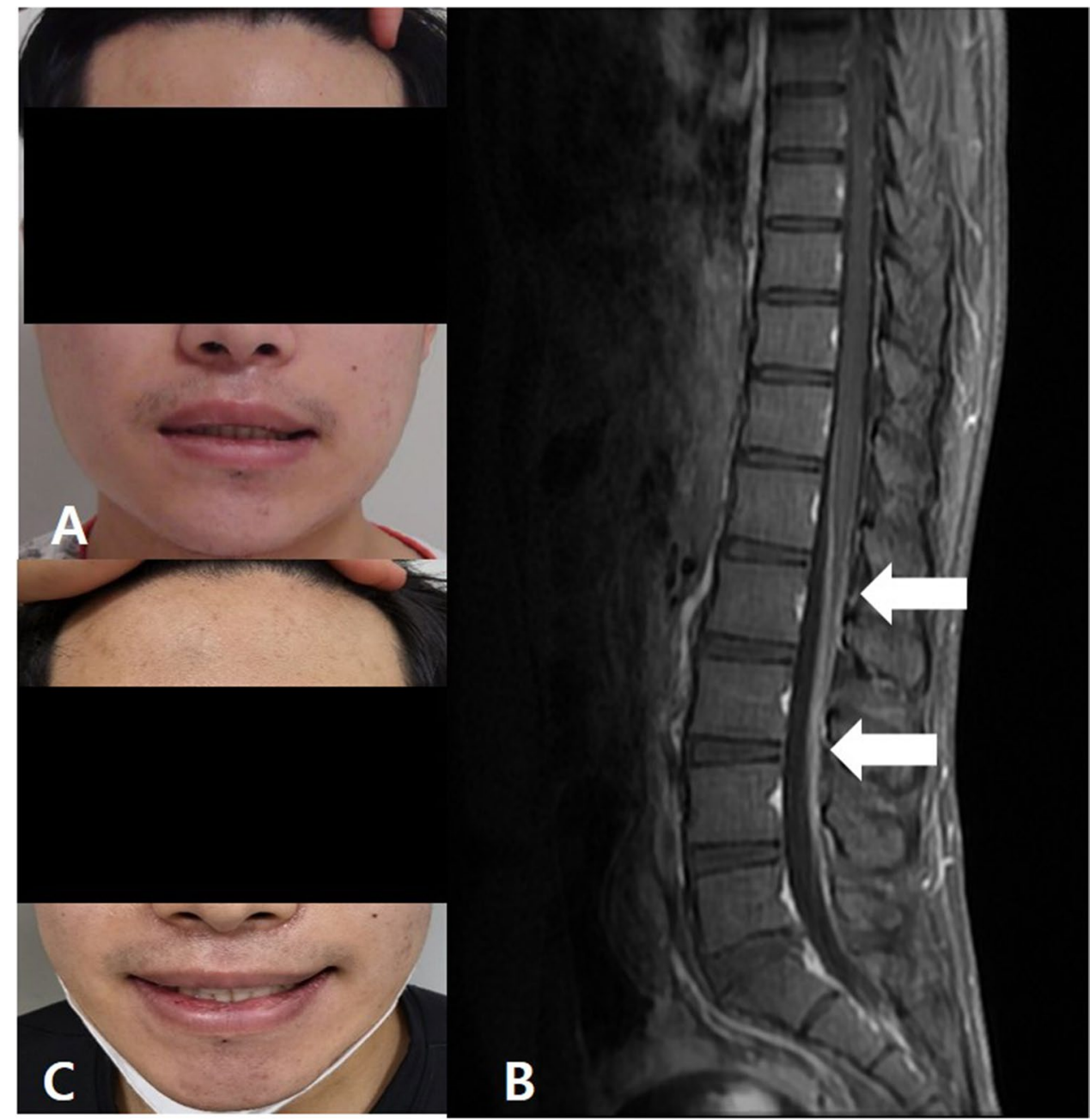

\& Johnson; ChAdox1nCoV-19, AstraZeneca) [1]. Recent studies have suggested a strong relationship between the vaccine and GBS, suggesting an autoimmune reaction triggered by immune responses that lead to the production of autoantibodies against proteins present in the myelin of peripheral nerves [2-7]. A systemic review illustrated the clinical characteristics of COVID-19 vaccine-associated GBS. According to the study, the mean time from vaccination to symptom onset was 11.3 days, with a mean age of 57.8 years and mild male predominance [7]. The initial manifestations are paresthesia, bilateral facial palsy, quadriparesis, myalgia, and dysautonomia, in descending order. Moreover, $>70 \%$ of the patients showed paresthesia, and $>60 \%$ presented with showed bilateral facial diplegia, as in our case. In addition, our patient showed dysautonomia that was also observed in other patients. Among the laboratory findings, $89 \%$ of the patients showed albuminocytologic dissociation. The first Pfizer vaccine-related GBS case showed a cauda equina enhancement [2], which was also observed in our patient who experienced urinary disturbances and erectile dysfunction. We believe that this case is closely related to GBS, as it showed a classical clinical manifestation of GBS without significant trigger factors. Moreover, our patient demonstrated most of the commonly observed features of GBS related to COVID-19 vaccine and showed favorable recovery after IVIg treatment. Another study showed the clinical manifestations of GBS associated with Pfizer vaccine only [4]. This study reviewed 19 similar cases reported till today, and only 5 of 19 patients manifested facial diplegia without significant muscle weakness, and this finding was different from a recent study reporting facial diplegia as one of the common features [7]. These clinical features appear to vary among studies, and this may be attributed to the limited number of patients enrolled or to an ethnic difference. Thus, more studies with a substantial number of patients are needed.

The underlying pathomechanism of COVID-19 vaccine and GBS is still unclear. However, patients appear to share common clinical symptoms with emphasis on significantly high frequency rate of facial diplegia compared to classical GBS. In conclusion, although GBS maybe a rare manifestation after COVID-19 vaccination, clinicians should 
recognize these neurological complications to provide early treatment.

\section{Declarations}

Conflict of interest The authors declare no competing interests.

Ethical approval and informed consent Informed and written consent was obtained from the patient.

\section{References}

1. Sharma O, Sultan AA, Ding H et al (2020) A review of the progress and challenges of developing a vaccine for COVID-19. Front Immunol 14(11):585354

2. Waheed S, Bayas A, Hindi F et al (2021) Neurological complications of COVID-19: Guillain-Barre syndrome following Pfizer COVID-19 vaccine. Cureus 18 13(2):e13426
3. Trimboli M, Zoleo P, Arabia G et al (2021) Guillain-Barré syndrome following BNT162b2 COVID-19 vaccine. Neurol Sci 42(11):4401-4402

4. Bouattour N, Hdiji O, Sakka S et al (2021) Guillain-Barré syndrome following the first dose of Pfizer-BioNTech COVID-19 vaccine: case report and review of reported cases. Neurol Sci $18: 1-7$

5. Nasuelli NA, De Marchi F, Cecchin M et al (2021) A case of acute demyelinating polyradiculoneuropathy with bilateral facial palsy after ChAdOx1 nCoV-19 vaccine. Neurol Sci 11:4747-4749

6. Kanabar G, Wilkinson P (2021) Guillain-Barré syndrome presenting with facial diplegia following COVID-19 vaccination in two patients. BMJ Case Rep 14(10):e244527

7. Shao SC, Wang CH, Chang KC et al (2021) Guillain-Barré syndrome associated with COVID-19 vaccination. Emerg Infect Dis $12: 3175-3178$

Publisher's note Springer Nature remains neutral with regard to jurisdictional claims in published maps and institutional affiliations. 